\section{Aportes del conocimiento de la ecoetología de los Trichomysterus (Actinopterygii, trichomycteridae) del río Umajalanta, Potosí, Bolivia.}
Enrique Richard ${ }^{1}$ y Denise Ilcen Contreras Zapata
${ }^{1}$ Escuela Superior Politécnica Agropecuaria de Manabí Manuel Félix López, Ecuador chelonos@gmail.com

\author{
Contribute to the ecoethology knowledge of \\ the Trichomysterus (Actinopterygii, \\ trichomycteridae) from the river \\ Umajalanta, Potosí, Bolivia.
}

Recibido: $17 / 06 / 2019$

Aceptado: 20/11/2019

Publicado: $27 / 12 / 2019$

\title{
RESUMEN
}

El objetivo del trabajo es contribuir al conocimiento de los aspectos ecoetológicos de los Trichomycterus del río Umajalanta. Estos bagres han sido citados con dos morfologías: una epigea, pigmentada ( $T$. cf barbouri), y alopátrica con la especie $T$. chaberti, hipogea, despigmentada, con visión reducida, endémica estricta y vulnerable. Se realizaron dos expediciones al área de estudio, por lo cual los morfos fueron estudiados in situ por observación directa, diseños experimentales etológicos y cámaras sumergidas con monitoreo por $24 \mathrm{~h}$. En este trabajo se demostró que: 1) Los Trichomycterus cf barbouri y T. chaberti son la misma especie. 2) Su hábitat es continuo y compartido. 3) El Trichomycterus sp es una especie hipogea facultativa con gran flexibilidad de adaptación ecológica. 4) Habita ambientes con temperaturas entre los $90^{\circ} \mathrm{C}$ y los $280^{\circ} \mathrm{C}$. 5) Los niveles de actividad varían en función de la temperatura, siendo más activos a temperaturas más altas. 6) Los individuos epigeos son preferentemente nocturnos, los hipogeos tienen actividad todo el día. 7) Pueden respirar aire atmosférico (respiración gástrica). 8) En condiciones de desecación de sus hábitats, la especie muestra la capacidad de desplazarse fuera del agua siguiendo líneas de humedad y sorteando pendientes de 460 a 750, en búsqueda de otros cuerpos de agua con mejores condiciones de habitabilidad. 9) Los individuos que viven en la oscuridad se ven despigmentados frente a una fuente de luz, pero cuando son iluminados durante varios minutos adoptan una coloración críptica con el entorno, revelando que sus funciones visuales son perfectamente funcionales.

PALABRAS CLAVE: adaptaciones, ecología, etología, hábitat, sinonimia.

\section{ABSTRACT}

The objective of the research is to contribute to the knowledge on the Eco ethologic of the Trichomycterus of Umajalanta river. This catfish has to morphologies: the pigmented epigeal ( $T$. cf barbouri), and the allopatric ( $T$. chaberti) hypogea depigmented, with reduced vision, endemic and vulnerable. Two expeditions to the study area have been made, so the morphs have been studied in situ thought direct observation, experimental ethological design and with $24 \mathrm{hrs}$ underwater camera recording. This work shows that: 1) The Trichomycterus cf barbouri and T. chabertiare are the same specie. 2) Their habitat is shared and continues. 3) The Trichomycterus $s p$ has a great ecological adaptation. 4) Lives in enviroments with temperatures between $90^{\circ} \mathrm{C}$ and $280^{\circ} \mathrm{C}$. 5) The activity levels vary according to temperature, been more 
actives at higher temperatures. 6) The epigeal specie are has activity during the night, the hypogea one activity is during the day. 7) They can breathe atmospheric air (gastric respiration). 8) In drought of their habitats the specie has the capacity to move out of the water and follow humidity lines looking for watered areas. 9) The individuals that live in the dark look depigmented at a light source, but when they are illuminated several minutes they acquire coloration according to the environment.

KEYWORDS: adaptation, ecology, ethology, habitat, synonymies.

\section{INTRODUCCIÓN}

La familia Trichomycteridae está formada por un grupo de bagres de tamaño pequeño a mediano, ampliamente distribuidos en la región Neotropical, desde Costa Rica hasta la Patagonia argentina, tanto en la vertiente atlántica como en la pacífica y, probablemente, la cordillera andina representa el área de mayor especiación (Ferraris, 2007).

El Trichomycterus es el género más diverso de la familia Trichomycteridae con más de 130 especies descriptas y muchas otras en espera de su descripción. La mayoría de las mismas son reofílicas, de hábitos generalmente crípticos y nocturnos. Habitan en ríos torrentosos de montaña (hasta $4500 \mathrm{msnm}$ ) y de tierras bajas, así como corrientes subterráneas (Trajano, 2001; Romero y Paulson, 2001).

En 1968, J. P. Durand describe, a partir de 30 ejemplares, una nueva especie de bagre (Trichomycterus chaberti) para la caverna de Umajalanta (Potosí, Bolivia), a los cuales refiere en su descripción original como "bagres blancos», sin embargo, luego en el mismo artículo indica que de los 30 ejemplares, 13 son medianamente pigmentados, 10 poco pigmentados $y$ siete serían despigmentados. También indica que el $50 \%$ de la muestra (15 ejemplares) tienen ojos de tamaño reducido. El mencionado autor señala que J. Chabert, el colector de los peces, no pudo ver otros peces fuera de la gruta, río arriba (Durand, 1968).

Una revisión exhaustiva desde 1968, fecha de la descripción del T. chaberti, a la actualidad (51 años), plantea la siguiente situación: para el río Umajalanta se ha descripto la existencia de dos especies de bagre (Trichomycteridae), una de las cuales, el Trichomycterus chaberti, se caracteriza como un pez blanco, despigmentado (Durand, 1968), o con pigmentación variable (Durand, 1968; Romero y Paulson 2001; Pouilly y Miranda 2003); ojos reducidos (Durand, 1968; Miranda 2000; Miranda y Pouilly 1999, 2003; Romero y Paulson 2001; Renno et al., 2007), o incluso no visibles externamente (Romero y Paulson 2001); lucífugos (Durand, 1968), hipogeo (Miranda, 2000; Miranda y Pouilly, 2009; Pouilly y Miranda, 2003; Renno et al., 2007; Do Nascimiento et al., 2001); troglobios (Miranda 1999; Pouilly y Miranda, 2003; Renno et al. 2007), restringido en su distribución a la caverna de Umajalanta (Pouilly y Miranda, 2001, 2003; Trajano, 2001; Renno et al., 2007); aislados geográficamente de otra especies de bagre (T. cf barbouri) (Miranda y Pouilly, 1999, 2001; Pouilly y Miranda, 2003; Renno et al., 2007), endémicos (Miranda y Pouilly, 1999; Pouilly y Miranda 2003; Renno et al., 2007) con estatus de conservación vulnerable a nivel nacional (Miranda y Pouilly, 2009) y de datos insuficientes a nivel internacional (Miranda y Pouilly, 2009); de mayor tamaño que su contraparte epigea (Miranda, 2000; Miranda y Pouilly, 1999; Pouilly y Miranda, 2003) entre otras diferencias (Miranda, 2000; Miranda y Pouilly, 2009; Pouilly y Miranda, 2003; Renno et al., 2007).

La otra especie es el Trichomycterus of barbouri, epígea, pigmentada y con marcadas diferencias morfológicas y ecológicas, con su contraparte hipogea, y de la cual también estaría 
geográficamente aislada, factor importante para argumentar una especiación alopátrica (Miranda y Pouilly, 1999, 2009; Pouilly y Miranda, 2001, 2003; Renno et al., 2007; Trajano, 2001). Algunos de estos datos fueron cuestionados previamente por Richard y Florentz (2017). Las dificultades inherentes de contextos hipogeos o espeleológicos obstaculizan en gran manera la exploración y estudios de la biodiversidad que caracteriza estos ecosistemas, en especial los orientados a la ecología y biología de las especies que allí viven (Trajano, 2001).

De aquí que tales estudios a nivel global sólo alcancen a menos del $4 \%$ frente a los estudios descriptivos, genéticos, etc. (Trajano, 2001). Dada la escasa información que se tiene de estos bagres habitantes de ambientes hipogeos, existe también la recomendación y necesidad de realizar estudios experimentales de Trichomycterus ex situ, especialmente dirigidos a caracterizar aspectos etológicos de los mismos (Renno et al., 2007). En función de lo expuesto, el objetivo del presente estudio es contribuir al conocimiento de los aspectos ecológicos y biológicos de los Trichomycterus que habitan en el río Umajalanta (porción epigea e hipogea), a partir de observaciones y estudios in situ y ex situ.

\section{MATERIALES Y MÉTODOS}

\section{Área de estudios.}

El río Umajalanta se ubica en el Parque Nacional Torotoro (Potosí, Bolivia), englobado en el Torotoro Geoparque Andino (Richard, 2017; Richard et al., 2018). La cabecera del Parque Nacional Torotoro se localiza en los Andes bolivianos ( $18^{\circ} 15^{\prime} \mathrm{S} ; 65^{\circ} 45^{\prime} \mathrm{W}$ ). La superficie del mismo tiene una altitud variable entre 1950 y $3850 \mathrm{~m}$.

Figura 1. Ubicación relativa del Torotoro Geoparque Andino y relieve del mismo.

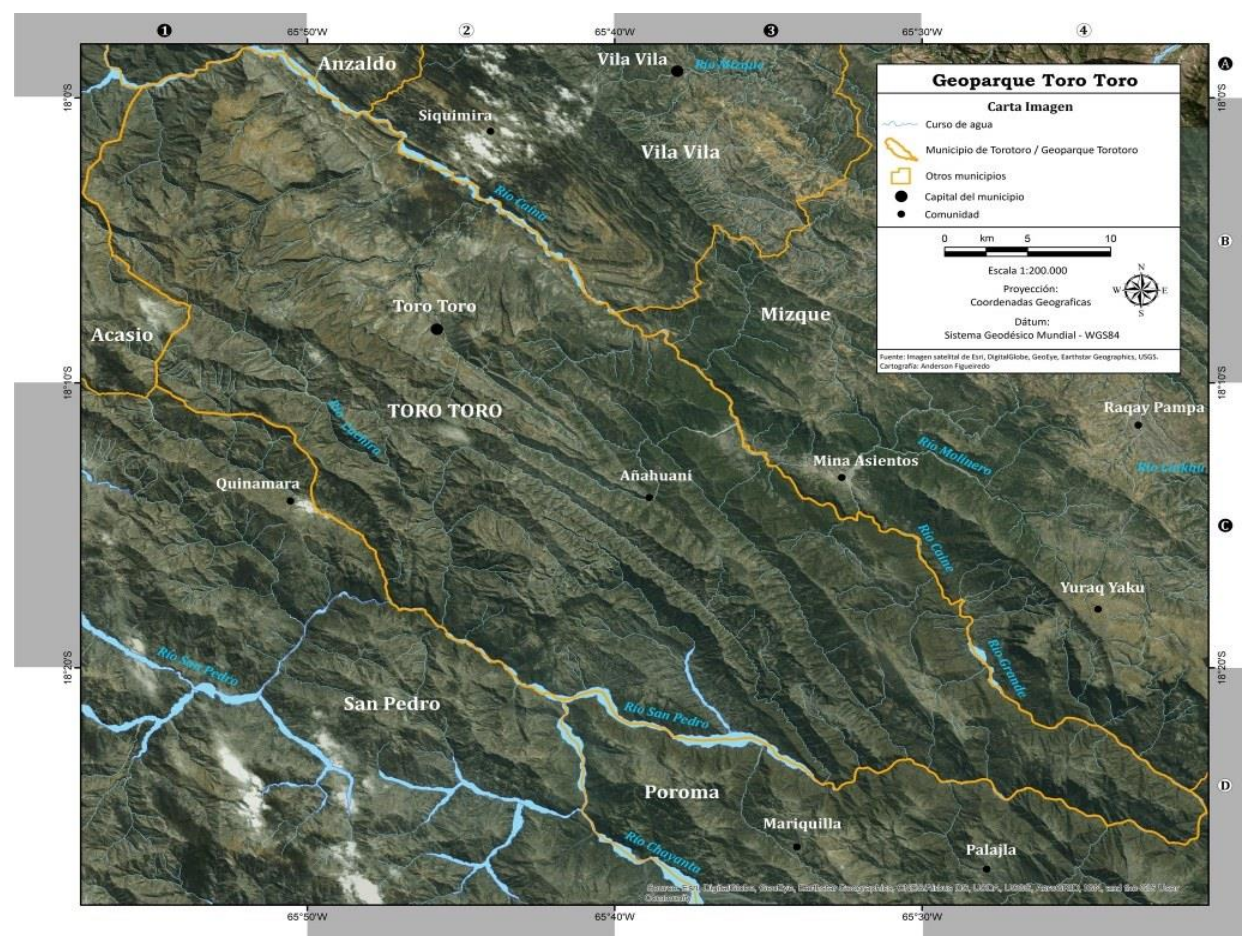

Fuente: Google Earth (2018).

El área corresponde mayormente a un pequeño sinclinal (45 por $9.5 \mathrm{~km}$ aprox.) (mapa 1), cuyos flancos laterales formados de estratos plegados casi verticales que, como consecuencia de la erosión, tomaron la forma de cerros laminares triangulares tipo hogbacks alineados, 
formando la estructura geomorfológica llamada de "Q'asa» (Menegat, 2017). Precisamente, uno de estos "Q'asa» se desplomó en algún período geológico de Torotoro sobre el río Umajalanta. La interacción del río por debajo de este "techo» o "Q'asa» formó la caverna de Umajalanta. Esta posee una amplia entrada de aproximadamente $30 \times 20 \mathrm{~m}$ (Latitud, $18^{\circ}$ 06'35"S; Longitud, 65⒋ $28^{\prime \prime} \mathrm{E} / 2830 \mathrm{~m}$ ) y el río Umajalanta recorre en su interior aproximadamente tres kilómetros para finalmente desembocar en el cañón de Torotoro desde una cascada de aproximadamente $20 \mathrm{~m}$.

Durante el año 2017 se realizaron dos expediciones científicas multidisciplinarias (agosto, septiembre y octubre) organizadas por la «Fundación emegece» y el Foro Latinoamericano de Ciencias Ambientales (FLACAM) y la Organización de las Naciones Unidas para la Educación, la Ciencia y la Cultura (UNESCO por sus siglas en inglés), a la región del Torotoro Geoparque Andino. Se eligió este período por ser la temporada seca y en la que los ríos presentan el menor caudal. Asimismo, el agua de los mismos es totalmente cristalina favoreciendo estudios in situ de su biodiversidad. En total se realizaron 27 días de observación in situ (174 h de observación acumuladas), tomando datos de 267 individuos epigeos (151 individuos) e hipogeos (116 individuos). Para ello se instalaron cuatro cámaras sumergibles Eken H9 en diferentes lugares del río Umajalanta, que cubrieron 24 h de observación acumulada (se debía reemplazar las baterías cada 120 minutos).

Luego, las cámaras eran cambiadas de sitio para una mayor cobertura de información. En total se documentó en video (Full HD 1920 x 1080 y 60 FPS y 30 FPS) ocho sitios de muestreo. También se utilizó una cámara Nikon 59700 y Nikon D3100 con lente Nikkor 55-300 mm para documentar observaciones fuera del agua. Para medir la inclinación de rocas se utilizó la aplicación «Clinometer» de (c) Plaincode para Android y sobre un terminal Samsung S4.

De igual forma los niveles de luz expresados en lux se midieron con la aplicación (c Samsung Galaxy Sensor sobre el mismo terminal. Se utilizó un termómetro químico de bulbo de mercurio para medir las temperaturas del aire y agua. La exploración de la caverna se realizó con equipo espeleológico estándar, linternas de cabeza con led (c) cree+95 de alta intensidad y lámparas led (C) Andoer de $1800 \mathrm{Lm}$ sumergible. En total se recorrieron $600 \mathrm{~m}$ en el interior de la caverna hasta llegar a la laguna Cachuela, que fue explorada usando equipo de buceo de apnea (traje de neoprene de $1 / 4$, máscara de silicona y snorkel) sin aletas para no enturbiar el agua.

En el interior de la laguna también se documentaron un total de ocho horas de video acumulados (en diferentes días), en resolución FullHD (1920 x 1080 px), lo cual permitió registrar el comportamiento de los bagres que la habitan. Los bagres estudiados fueron capturados con redes marca Tetra para peceras con malla de $7 \times 8 \mathrm{~cm}$ y mango de $22 \mathrm{~cm}$ y otra de 13 × 25 y mango de $35 \mathrm{~cm}$. Para el conteo de individuos y eventos se utilizó un cuentaganado manual de cuatro dígitos.

La comprobación de la conexión y flujo continuo del agua entre las diferentes cámaras de la caverna se realizó con el empleo de un total de 12 esferas de poliestireno expandido, de $3 \mathrm{~cm}$ de diámetro y de color amarillo fluorescente desde la entrada de la misma. Para las mediciones de los bagres se utilizó un calibre estándar. Con autorización del Servicio Nacional de Áreas Protegidas de Bolivia y la Alcaldía Municipal de Torotoro se capturó un total de 12 ejemplares ( 6 epigeos y 6 hipogeos) para su estudio ex situ, durante un año en peceras de $30 \mathrm{x}$ $40 \times 80 \mathrm{~cm}$. Concluido el estudio los bagres fueron devueltos al lugar de origen.

Complementariamente se realizó un total de 134 encuestas a los pobladores, a fin de evaluar el conocimiento de la población local sobre la biología, ecología, usos de estos peces; así como 
de los potenciales impactos de las actividades humanas sobre el río Umajalanta. Finalmente, se hizo un análisis exhaustivo de toda la bibliografía disponible sobre el tema, incluido informes, tesis no publicadas, etc., desde 1968, fecha de descripción de $T$. chaberti, hasta la actualidad.

\section{RESULTADOS Y DISCUSIÓN}

Como resultado del estudio se pudo explorar el recorrido exterior del río Umajalanta desde las cercanías de su naciente hasta el interior de la caverna de Umajalanta, incluyendo la laguna Cachuela, mejor conocida como laguna de los bagres «ciegos» como sendero turístico del lugar (Richard, 2017). En este recorrido (figura 2) y período de estudio se pudo confirmar, entre otros aspectos, que los bagres con morfología de Trichomycterus cf barbouri y de $T$. chaberti fueron hallados indistintamente tanto en la parte epigea como hipogea del río Umajalanta.

Figura 2. Esquema de planta indicando el recorrido del río Umajalanta en el interior de la caverna homónima.

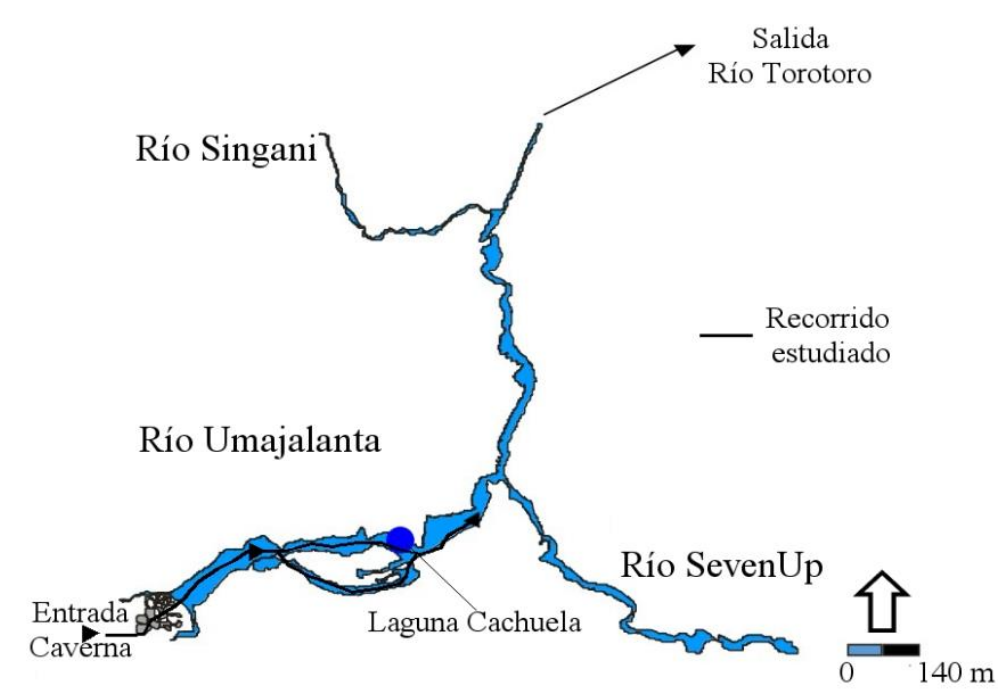

Nota: El trazo negro continuo indica el recorrido realizado en este estudio. La flecha negra la dirección del flujo de agua. Fuente: Elaboración propia.

El río, tanto en su parte epigea como hipogea posee una inclinación que en algunos trayectos supera los $45^{\circ}$ y numerosas cascadas entre uno y tres metros de altura entre cuerpos de agua. En 12 pequeñas lagunas epigeas (no mayores de tres metros de diámetro y aprox. $70 \mathrm{~cm}$ de profundidad) se hallaron ambas morfologías. Si bien se ha afirmado en varios artículos (Miranda y Pouilly, 1999; Miranda y Pouilly, 2001; Pouilly y Miranda, 2003; Renno et al., 2007) que las poblaciones epigeas e hipogeas están aisladas al flujo genético, la presente investigación demuestra lo contrario.

Los bagres epigeos llegan al interior de la caverna y laguna de Cachuela junto a otros elementos de la biodiversidad epigea como larvas de anfibios (Boana alboniger y otras especies sin identificar), larvas de insectos (identificados dentro de los órdenes Odonata, Ephemeroptera, Trichoptera, Plecopteras y Coleoptera y Hemiptera), así como las pelotas de polipropileno expandido, de las cuales se rescataron cuatro en la laguna Cachuela y el resto en diferentes cuerpos de agua interconectados. Consecuentemente, no existe aislamiento geográfico o geomorfológico entre las poblaciones epigeas e hipogeas y, por tanto, el flujo genético es potencialmente viable desde el exterior de la caverna hacia el interior. 
A la inversa, posiblemente sea más dificultoso, en particular por tener que nadar a contracorriente en época seca por la presencia de cascadas y escaso volumen de agua. Sin embargo, una de las características frecuentes y reportadas para el género Trichomycterus es precisamente la habilidad de subir cascadas soportando fuertes corrientes (reotaxis positiva) (Barbosa y Costa, 2012; Sleen y Albert, 2017) y acorde a lo aquí estudiado y documentado por primera vez para la especie, los Trichomycterus del río Umajalanta no son la excepción.

Los adultos de la especie ( $n=12,6$ epigeos y 6 hipogeos) son sensibles al nivel del agua y la temperatura de la misma. Al colocar ejemplares individuales o en pares sobre una concavidad de una roca aledaña al río, se observó que cuando el nivel del agua es inferior a tres o cuatro centímetros o la temperatura supera los $28^{\circ} \mathrm{C}$, el animal abandona el cuerpo de agua siguiendo preferentemente los rastros de agua y trepando pendientes de $46^{\circ}$ a $75^{\circ}$, o sorteando obstáculos de hasta siete centímetros a $90^{\circ}$ en la búsqueda de otros cuerpos de agua (figura 3)

Figura 3. Ejemplar epigeo saliendo del agua y trepando una pendiente de roca de $46^{\circ}$ siguiendo una línea de humedad.

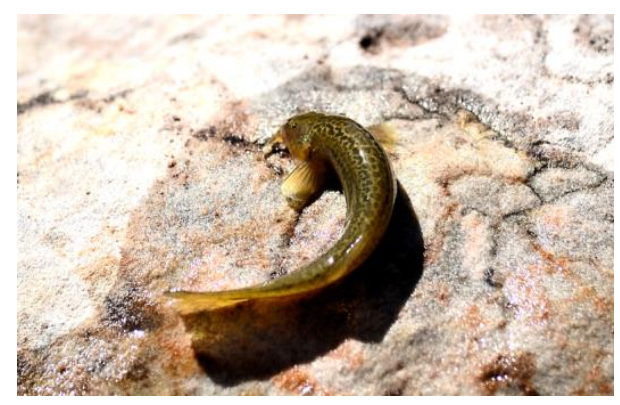

Fuente: E. Richard
Figura 4: Ejemplar hipogeo mostrando el primer radio de la aleta pectoral en forma de gancho, uno de los elementos usados para trepar pendientes.

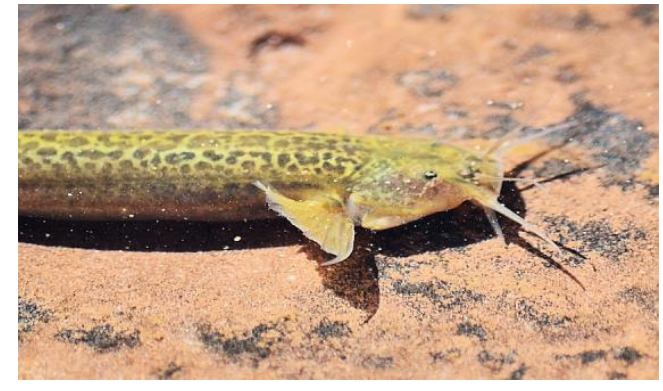

Fuente: E. Richard

Probablemente (hipótesis planteada), este comportamiento observado in situ se debe a que, en temporada seca, muchos cuerpos de agua donde habitan quedan reducidos o incluso secos, como se ha observado en este estudio. En este caso, el poder "caminar» o "trepar» usando ondulaciones de su cuerpo, las placas ventrales del hueso opercular e interopercular, rellenas de numerosos odontotos y unidos por un ligamento robusto, así como las aletas pectorales con su primer radio prolongado como "ganchos», sin duda ayudan a la especie a evitar la muerte encontrando otros cuerpos de agua (figura 4).

Por otro lado, si bien el río Umajalanta en todo su trayecto, por la inclinación y el relativo número de cascadas, posee aguas muy oxigenadas, se observó y filmó in situ ( $\mathrm{n}=5$ forma hipogea, $n=11$ forma epigea) y ex situ ( $n=342$ forma hipogea, $n=345$ forma epigea), que los individuos periódicamente suben a la superficie a tomar aire con fines respiratorios. La respiración atmosférica gástrica es también una característica de muchos Siluriformes (Graham, 1997) y Trichomycteridae (Romero, 1987; Rapp, 2000; Sleen y Albert, 2017, y con la presente investigación se documentó por primera vez para la especie (figuras $5 a$ y $5 b$ ). Sin duda, esta aptitud, junto a la de poder «caminar» o "trepar» le ayudaría mucho en la supervivencia en condiciones de sequedad de los cuerpos de agua donde habita y que en invierno algunos quedan incomunicados entre sí.

Figura 5a. Individuo de la forma epigea emergiendo a tomar aire.
Figura 5b. Mismo individuo retronando al fondo luego de tragar una bocanada de aire 
atmosférico.

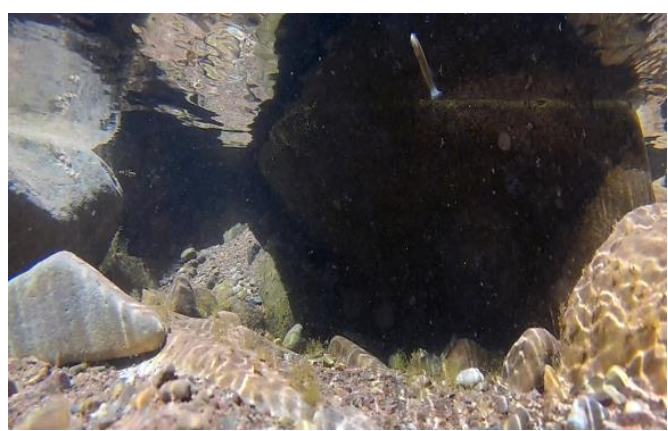

Fuente: E. Richard

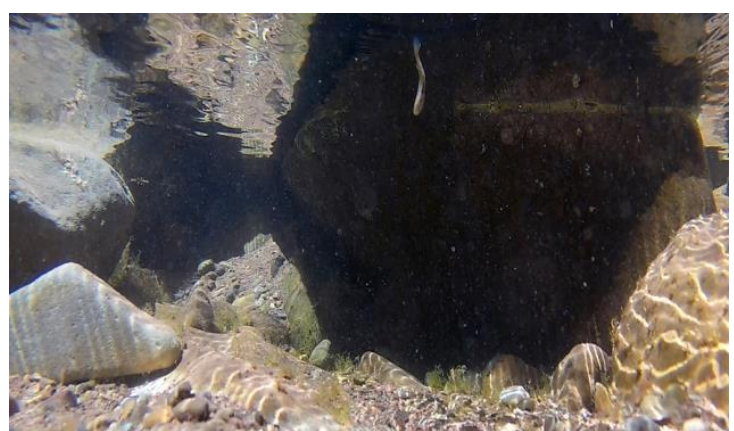

Fuente: E. Richard

En época de verano el caudal del río aumenta exponencialmente, con lo cual adquiere una continuidad de caudal ininterrumpido y se hace más fácil, para las formas hipogeas, remontar corriente arriba usando la reotaxis positiva que poseen, así como las habilidades aquí documentadas para trepar rocas. De cualquier forma, el flujo genético en una dirección u otra, incluso en ambas se demuestra que es un hecho innegable.

Lo anterior, sumado al estudio genético de Renno et al. (2007), en el cual se indica que las diferencias genéticas (variabilidad alozimica) son casi nulas entre ejemplares hipogeos y epigeos, permite afirmar que los Trichomycterus del río Umajalanta pertenecen a la misma especie (sinonimia en estudio). Esto ya fue predicho en su momento por el Dr. Ringuelet (1974), cuyo trabajo no ha sido citado en ningún estudio publicado sobre los Trichomycterus de Umajalanta posterior a su publicación, al menos como lo demuestra la revisión bibliográfica realizada por los autores del presente trabajo. Asimismo, no se niega la posibilidad de que otros autores aquí referenciados (Miranda, 2000; Miranda y Pouilly, 2001; Pouilly y Miranda, 2003) hayan encontrado diferencias morfológicas, incluso significativas, entre los bagres epigeos e hipogeos; como tampoco se cuestiona el hecho de que estas características se deban a la plasticidad fenotípica que manifiesta el género Trichomycterus (Trajano, 2001; Rapp, 2000; Romero y Poulson, 2001; Romero y Green, 2005).

De igual forma, las diferencias etológicas y ecológicas in situ solo indicarían la facilidad adaptativa fenotípica de la especie a condiciones cambiantes, otra característica de los Trichomycterus (Trajano, 2001; Rapp, 2000; Romero y Poulson, 2001; Romero y Green. 2005). En este sentido, el río Umajalanta es un ambiente lótico con cascadas, pero continuo (figura 2) al menos en la temporada de verano, y dicha continuidad se expresa en la parte epigea como hipogea. Se debe tener en cuenta también que la caverna solo es el resultado de una caída de un " $Q$ 'asa» que formó un techo sobre el cauce que continua sin interrupciones hasta la desembocadura, en el río Torotoro, por lo cual el flujo de peces en el sentido de la corriente es ininterrumpido y en sentido contrario no es imposible como se ha argumentado. 
Figura 6a. Dos ejemplares de la forma hipogea fotografiado in situ (laguna "Cachuela»).

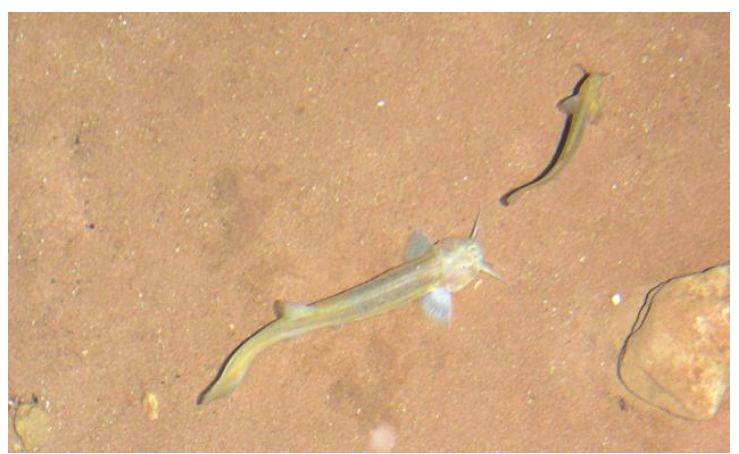

Figura 6b. Ejemplar de la forma epigea fotografiado in situ y mostrando su característica coloración críptica.

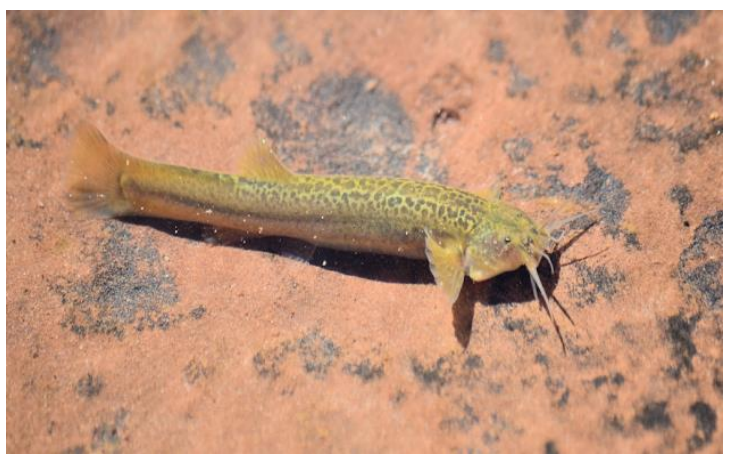

Figura 6c. En la caja de Petri, formas hipogea y epigea luego de estar cerca de 10 minutos a la luz del sol (El más pequeño hipogeo, el otro epigeo).

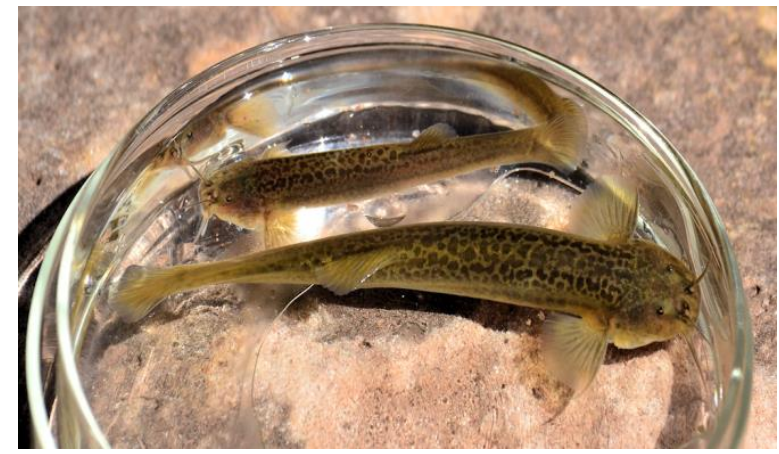

Fuente: E. Richard

Por lo tanto, las formas epigeas e hipogeas no solo serían la misma especie, sino que tampoco cumplen con las características de una especie troglobia (Trajano, 2001), por lo cual la misma debería ser considerada solo como especie troglofila (Trajano, 2001). En la parte epigea los bagres adultos muestran un comportamiento extraordinariamente críptico, propio de una mayor profundidad posible y siempre bajo la sombra de las rocas ( $n=84,55,62 \%$ ) (Escotofilia, fide Romero, 1987). Sólo salieron eventualmente en busca de alimentos individuos juveniles (Aprox. de tres a cinco centímetros) $(n=28,18,54 \%)$, los cuales al parecer son más propensos a esta actividad diurna que los adultos.

Cuando un bagre adulto es obligado a salir de abajo de las rocas normalmente está descolorido con un color crema uniforme o con una coloración gris azulina clara $(n=98,64,9 \%)$; pero al ser capturados y expuestos a la luz solar en un frasco de vidrio, a los pocos minutos adoptan la típica coloración críptica de cuerpo con manchas color castaño (Figuras $6 a, b$ y c). A lo largo del río Umajalanta, tanto en la parte epigea como hipogea, se hallaron bagres que respondían a la descripción de $T$. cf barbouri como de T. chaberti indistintamente. Cabe destacar que, acorde a Durand (1968), los exploradores que descubrieron los bagres hipogeos no hallaron bagres en la parte epigea del río, probablemente debido al comportamiento críptico aquí descripto.

A partir de las $19 \mathrm{~h}$ aproximadamente (período de estudio) comienzan a salir los bagres de la parte epigea e inician su actividad trófica, la cual se mantiene a lo largo de toda la noche y hasta la salida del sol. En este período se observaron indistintamente bagres con coloración criptica y bagres con coloración uniforme crema, castaño claro (beige), gris y gris azulado. En 
ningún caso se observaron ejemplares con ojos de diferente tamaño. En toda la extensión recorrida del río Umajalanta, en el período de estudio, se constató además una gran variabilidad de temperaturas en los cuerpos de agua donde fueron hallados los bagres. Así, en la parte epigea del río, los valores oscilaron entre los $24^{\circ} \mathrm{C}$ máximo (aprox. a $400 \mathrm{~m}$ de la entrada de la caverna) y $12^{\circ} \mathrm{C}$ mínimo (sobre la entrada de esta); en tanto que en la parte hipogea el valor máximo fue de $12^{\circ} \mathrm{C}$ en la entrada, $9^{\circ} \mathrm{C}$ en la superficie de la Laguna y $8^{\circ} \mathrm{C}$ en el fondo de la misma.

Los bagres epigeos se hallaron tanto en lechos rocosos y sin vegetación como en lechos arenosos y limosos, con vegetación de alga densa. Los hipogeos se encontraron en lechos rocosos y arenosos indistintamente. En la parte hipogea del río desde la entrada y a lo largo de $600 \mathrm{~m}$ de recorrido interno dentro de la caverna, se hallaron bagres tanto en el río mismo como en las pequeñas lagunitas o pozos de agua formados por las irregularidades rocosas del cauce del río. En los primeros 40 metros donde todavía llega la luz del sol, los bagres muestran el mismo comportamiento críptico (escondidos bajos las rocas y con poca actividad) que los de la parte epigea.

Por otro lado, los bagres de la caverna donde ya no llega la luz solar (0 lux) manifiestan actividad trófica durante las $24 \mathrm{~h}$ y no muestran un comportamiento críptico o lucifugo evidente, pero son comparativamente más lentos (Richard y Florentz 2017), al parecer, por la baja temperatura del agua en esta parte del río. Asimismo, al ser iluminados con la lámpara led, en ningún caso $(n=116,100 \%)$ se mostraron lucífugos como lo indica Durand (1968) en su trabajo. Por otro lado, en la laguna Cachuela se observaron bagres con coloración críptica, crema, castaño claro, gris, gris azulado y de igual forma a lo comentado para la parte epigea. Muchos de ellos frente a la luz adoptan coloración críptica.

Miranda y Pouilly $(2001,2003)$ también señalan que los bagres «descoloridos» de la porción hipogea del río al ser expuestos a una luz adquieren a los pocos minutos la coloración críptica aquí descripta. Una muestra de seis bagres capturados al azar en la laguna Cachuela y llevados a la superficie, luego de ser expuestos en un frasco entre unos cinco y ocho minutos a la luz del sol, adquirieron la misma coloración críptica que otro grupo de seis bagres epigeos (muestra testigo), haciéndose indistinguibles unos de los otros (figuras $6 a, b$ yc).

Richard y Florentz (2017) indican que en los bagres de la laguna Cachuela no se observaron diferencias significativas en el tamaño de los ojos, ni tampoco en cuanto a su funcionalidad, pues al recibir estímulos lumínicos cambian el color de su piel al patrón críptico. Asimismo, se observó durante el buceo y filmación subacuática que mueven sus ojos independientemente como lo hacen sus contrapartes epigeas. Cabe destacar que los autores (Miranda, 2000; Miranda y Pouilly, 2001; Pouilly y Miranda, 2003; Miranda y Pouilly, 2009) que sostienen que los individuos hipogeos ( $T$. chaberti) son una especie diferente a la epigea ( $T$. cf barbouri) indican, paradójicamente en sus estudios, que los individuos hipogeos poseen incluso mayor cantidad de cromatóforos que los epigeos. De igual forma estos autores no encontraron diferencias en el tamaño de las barbillas como era de esperarse frente a una adaptación al medio hipogeo.

\section{CONCLUSIONES}

Debido a que: 1) No hay sustento alguno para fundamentar un aislamiento geográfico y por tanto genético entre los bagres hipogeos y epigeos. 2) Existe un estudio (Renno et al., 2007) que indica que las diferencias genéticas (variabilidad alozimica) son casi nulas entre ejemplares hipogeos y epigeos 3) Etológicamente, el estudio durante un año de ambas muestras, epigeas e hipogeas, en acuarios con las mismas características y condiciones no mostró diferencias 
etológicas entre ellas 4) Las morfologías aquí observadas entre los individuos hipogeos y epigeos son muy similares y poco significativas. 5) La mayor parte de las diferencias ecológicas y etológicas observadas in situ se explican en función al contexto en el que se desenvuelven las diferentes poblaciones de Trichomycterus; se concluye, acorde a las evidencias ecológicas y etológicas estudiadas in situ, ex situ y el análisis de la bibliografía pertinente, que las formas epigeas e hipogeas pertenecen a una misma especie (sinonimia en estudio) y que de ninguna manera puede seguir considerándose troglobia; pues la evidencia la indica más apropiadamente como troglofila.

Asimismo, se registra que las diferencias morfológicas y de coloración observadas por algunos autores, sin duda se deben a la plasticidad fenotípica que manifiesta el género Trichomycterus. De igual forma, las diferencias etológicas y ecológicas in situ solo indican la plasticidad adaptativa de la especie a condiciones cambiantes, otra característica de los Trichomycterus.

\section{REFERENCIAS BIBLIOGRÁFICAS}

Barbosa, M. y Costa, W. (2012) Trichomycterus puriventris (Teleostei: Siluriformes: Trichomycteridae), a new species of catfish from the río Paraíba do Sul basin, southeastern Brazil. Vertebrate Zoology, 62(2), 155 - 160.

Do Nascimiento, C., Villareal O. y Provenzano F. (2001). Descripción de una nueva especie de bagre anoftalmo del género Trichomycterus (Siluriformes, Trichomycteridae) de una cueva de la sierra de Perijá, Venezuela. Bol. Soc. Venezolana Espel., (35), 20-28.

Durand, J. (1968). Etude des poissons récoltés dans la grotte de Umayalanta (Bolivie), Trichomycterus chaberti sp. n. Ann. Spéléol, 23(2): 343-353.

Ferraris, C.J.Jr. (2007). Checklist of catfishes, recent and fossil (Osteichthyes: Siluriformes), and catalogue of siluriform primary types. Zootaxa,1418(1), 1-628. http://dx.doi.org/10.11646/zootaxa.1418.1.1

Graham, J. (1997). Air breathing Fishes: Evolution, Diversity, and Adaptation. Academic Press.

Menegat, R. (2017). Geoparque Torotoro: la memoria entera de un mar fósil que se transformó en montaña. Curso Internacional: Proyección del geoparque Torotoro, Potosí, Bolivia.

Miranda, G. (2000). Adaptaciones biológicas y ecológicas de peces del género Trichomycterus al ambiente cavernícola en el Parque Nacional Torotoro [Tesis de licenciatura no publicada]. Universidad Mayor de San Andrés, Bolivia.

Miranda, G. y Pouilly, M. (1999) Ecología comparativa de poblaciones superficiales y cavernícolas de Trichomycterus spp (Siluriformes) en el Parque Nacional Torotoro. Revista Boliviana de Ecología y Conservación Ambiental, (6), 163-171. https://cutt.ly/GyK9kQd

Miranda, G. y Pouilly M. (2001, 15 al 22 de junio). Morphology and reproductive strategies of cave fish of genus Trichomycterus in Torotoro National Park (Potosí, Bolivia). En Speleo Brazil 2001. https://cutt.ly/EyK3keC

Miranda, G. y Pouilly, M. (2009). Trichomycterus chaberti en L.F. Aguirre, R. Aguayo, J. Balderrama, C. Cortez y T. Tarifa, Libro rojo de la fauna silvestre de vertebrados de Bolivia (pp. 77-78). Ministerio de Medio Ambiente y Agua. 
Pouilly, M. y Miranda, G. (2003). Morphology and reproduction of the cavefish Trichomycterus chaberti and the related epigean Trichomycterus cf. barbouri. Journal of Fish Biology, 63, 490 - 505. https://doi.org/10.1046/j.1095-8649.2003.00171.x

Rapp, P. y Daniel, L. H. (2000, 20-23 July). Tracking evolutionary trends in Siluriformes (Teleostei: Ostaríophysi) [Paper]. In: International Congress on the Biology of Fish. Evolution Symposium Proceedings. Scotland, UK, 57-72.

Renno, J. F., Gazel, C., Miranda, G., Pouilly, M. y Berrebi, P. (2007). Delimiting species by reproductive isolation: the genetic strucutre of epigean and hypogean Trichomycterus spp. (Teleostei, Siluriformes) in the restricted área of Torotoro (Upper Amazon, Bolivia). Genetica, 131(3), 325-336. https://doi.org/10.1007/s10709-007-9142-9

Richard, E. (2017). Sobre el estado actual los bagres (Trichomycterus chaberti, Actinopterygii, Siluriformes) en tres cuerpos de agua de la caverna de Umajalanta (Geoparque Andino Torotoro, Potosí, Bolivia) y potenciales amenazas. Rev. NLDE Fund. Emegece, 3(2), 12.

Richard, E. y Florentz, M. (2017). Nuevos datos y descubrimientos sobre la historia natural del bagre de Umajalanta (¿Trichomycterus chaberti?) de la III Misión Científica de la Fundación emegece a Torotoro (Potosí, Bolivia). Rev. NLDE Fund. emegece 3(6), 10 -11.

Richard, E., García, G. y Contreras, D. (2018). Geoparques: Lugar de encuentro para la geofilia, biofilia, cultura de la contemplación y turismo especializado y científico, el caso del Torotoro Geoparque Andino (Potosí, Bolivia) en M.A. Díaz Ponce et al. (Eds.), Dossier académico: Bosques, recursos naturales y turismo sostenible (pp.12-28). Editorial CIDE.

Ringuelet, R. (1974). Breves comentarios sobre un pez cavernícola de Bolivia (Pygidium chaberti Durand, 1968). Neotropica, 20(62), 65-66.

Romero, A.Jr. (1987). Observaciones preliminares sobre la conducta en individuos cavernícolas de Trichomycterus conradi (Pisces: Trichomycteridae). Boletín de la Sociedad Venezolana de Ciencias Naturales, 41(144), 205-216. https://cutt.ly/0yK3Mlr

Romero, A. y Poulson, K. (2001). It's a wonderful hipogean life: a guide to the troglomorphic fishes of the world. Environmental Biology of fishes, 62, 13-41. https://doi.org/10.1023/A:1011844404235

Romero, A. y Green, S.M. (2005). The end of regressive evolution: examining and interpreting the evidence from cave fishes. Journal of Fish Biology,67(1), 3-32. https://doi.org/10.1111/i.0022-1112.2005.00776.x

Sleen, P. y Albert, J.S. (Eds). (2017). Field Guide to the Fishes of the Amazon, Orinoco, and Guianas. Princeton University Press.

Trajano, E. (2001). Ecology of subterranean fishes: an overview. Environmental Biology of Fishes, 62, 133-160. https://doi.org/10.1023/A:1011841913569 